\title{
Position of Transmembrane Helix 6 Determines Receptor G Protein Coupling Specificity
}

\author{
Alexander S. Rose, ${ }^{\dagger,}$ Matthias Elgeti, ${ }^{\dagger, \nabla}$ Ulrich Zachariae, ${ }^{\S}$ Helmut Grubmüller, $"$ Klaus Peter Hofmann, ${ }^{\dagger, \perp}$ \\ Patrick Scheerer, ${ }^{\dagger, \#}$ and Peter W. Hildebrand ${ }^{*}, \dagger, \ddagger$ \\ ${ }^{\dagger}$ Institut für Medizinische Physik und Biophysik, ${ }^{\ddagger}$ AG ProteiInformatics, and ${ }^{\#}$ AG Protein X-ray Crystallography, \\ Charité-Universitätsmedizin Berlin, Charitéplatz 1, 10117 Berlin, Germany \\ ${ }^{\S}$ Division of Computational Biology, College of Life Sciences, and Division of Physics, School of Engineering, Physics and \\ Mathematics, University of Dundee, Dow Street, Dundee DD1 5EH, U.K. \\ "Department of Theoretical and Computational Biophysics, Max-Planck-Institute for Biophysical Chemistry, D-37077 Göttingen, \\ Germany \\ ${ }^{\perp}$ Centre of Biophysics and Bioinformatics, Humboldt-Universität zu Berlin, Invalidenstrasse 42, D-10115 Berlin, Germany
}

Supporting Information

ABSTRACT: G protein coupled receptors (GPCRs) transmit extracellular signals into the cell by binding and activating different intracellular signaling proteins, such as G proteins ( $\mathrm{G} \alpha \beta \gamma$, families $\mathrm{Gi}, \mathrm{Gs}, \mathrm{Gq}, \mathrm{G}_{12 / 13}$ ) or arrestins. To address the issue of Gs vs Gi coupling specificity, we carried out molecular dynamics simulations of lipidembedded active $\beta_{2}$-adrenoceptor $\left(\beta_{2} \mathrm{AR}^{*}\right)$ in complex with $\mathrm{C}$-terminal peptides derived from the key interaction site of $\mathrm{G} \alpha(\mathrm{G} \alpha \mathrm{CT})$ as surrogate of $\mathrm{G} \alpha \beta \gamma$. We find that $\mathrm{Gi} \alpha \mathrm{CT}$ and $\mathrm{Gs} \alpha \mathrm{CT}$ exploit distinct cytoplasmic receptor conformations that coexist in the uncomplexed $\beta_{2} \mathrm{AR}^{*}$. The slim $\mathrm{Gi} \alpha \mathrm{CT}$ stabilizes a $\beta_{2} \mathrm{AR}^{*}$ conformation, not accessible to the bulkier Gs $\alpha \mathrm{CT}$, which requires a larger TM6 outward tilt for binding. Our results suggest that the TM6 conformational heterogeneity regulates the catalytic activity of $\beta_{2} \mathrm{AR}^{*}$ toward Gi or Gs.

$G$ protein coupled receptors (GPCRs) transduce a large

variety of extracellular signals into the cell. One and the same receptor can activate different intracellular downstream signaling proteins such as heterotrimeric $\mathrm{G}$ proteins $(\mathrm{G} \alpha \beta \gamma$, families $\mathrm{Gi}, \mathrm{Gs}, \mathrm{Gq}, \mathrm{G}_{12 / 13}$ ) or arrestins (arrestin 1-4), resulting in different cellular and physiological responses. ${ }^{1}$ Understanding the molecular mechanism of this coupling promiscuity is therefore one of the key questions in current receptor research. The phenomenon that ligands can bias toward arrestin- or G protein-specific signaling, respectively, has been linked to the existence of different conformations of the active receptor $\left(\mathrm{R}^{*}\right)$, with different $\mathrm{G}$ protein and arrestin binding properties. ${ }^{2}$ The first structural evidence for how different ligands bias the human $\beta_{2}$-adrenoceptor $\left(\beta_{2} \mathrm{AR}\right)$ toward arrestin or $G$ protein signaling has recently been provided. ${ }^{3}$ However, little is known about the structural mechanism by which receptors select between different $G$ protein signaling pathways. ${ }^{4}$

In this study we performed classical all-atom molecular dynamics (MD) simulations to investigate how the conformational space of the active human $\beta_{2} \mathrm{AR}^{*}$ is exploited for selective coupling to Gs or Gi. For comparison we chose bovine rhodopsin (RhR), which is capable of interaction with only a single $G$ protein, namely $G t$, a member of the Gi family. Our report will focus on the conformational heterogeneity of transmembrane helix 6 (TM6), which is released from the 7TM helix bundle upon activation, resulting in the prominent outward tilt ${ }^{5}$ observed in crystal structures of active GPCRs. ${ }^{6-9}$ This hallmark event of GPCR activation leads to the formation of a highly flexible cytoplasmic crevice $\mathrm{e}^{10-12}$ to which the Cterminus of the $\mathrm{G} \alpha$ subunit $(\mathrm{G} \alpha \mathrm{CT}){ }^{6,7,13,14}$ a key determinant of $\mathrm{G}$ protein coupling specificity, ${ }^{15}$ binds. Our present analysis suggests that distinct TM6 outward tilts are responsible for Giand Gs-specific signaling by $\beta_{2} \mathrm{AR}^{*}$.

So far, the only crystal structure of a $R^{*} \cdot G$ complex reported is that of $\beta_{2} \mathrm{AR}^{*}$ bound to Gs. ${ }^{7}$ Comparison with crystal structures of active rhodopsin (RhR*) in complex with Gt $\alpha \mathrm{CT}^{6,16,17}$ reveals that both G $\alpha$ C-termini adopt an $\alpha$-helical conformation terminated by a reverse turn. Gs $\alpha \mathrm{CT}$ and Gt $\alpha \mathrm{CT}$ bind with the tip of the reverse turn to $R^{3.50}$ (BallesterosWeinstein nomenclature) from the E(D)RY motif at the base of the cytoplasmic crevice of $\mathrm{R}^{*}$. However, the reverse turn of $\mathrm{Gs} \alpha \mathrm{CT}$ is bulkier than those of $\mathrm{Gt} \alpha \mathrm{CT}$ and its close homologue Gi $\alpha \mathrm{CT}$ (Figure S9). Specifically, the cation $-\pi$ interaction between $\mathrm{Y} 391$ and $\mathrm{R} 131^{3.50}$ in the $\beta_{2} \mathrm{AR} * \mathrm{Gs}$ complex seems to require a 5-6 Å larger TM6 outward tilt than the hydrogen bond between the carbonyl oxygen of C347 and the guanidinium group of $\mathrm{R} 135^{3.50}$ in $\mathrm{RhR}^{*} \cdot \mathrm{Gt} \alpha \mathrm{CT}^{6}$ (Figure $1 \mathrm{~A}, \mathrm{~B})$. The present study was thus motivated by the idea that the different space requirements for the key interactions of Gt $\alpha \mathrm{CT}$ and Gs $\alpha \mathrm{CT}$ with $\mathrm{R}^{*}$ result in distinct TM6 outward tilts and differently shaped cytoplasmic crevices in the corresponding complexes (Figure 1C,D).

To find out whether a $\mathrm{RhR}^{*}$-like conformation exists in $\beta_{2} \mathrm{AR}^{*}$, which might be stabilized by $\mathrm{Gi} \alpha \mathrm{CT}$ and thus be responsible for selective signaling through $\mathrm{Gi}$, a series of $\mathrm{MD}$ simulations of the uncomplexed $\beta_{2} \mathrm{AR}^{*}$ was started using the receptor coordinates from the $\beta_{2} \mathrm{AR}^{*}$.Gs complex. ${ }^{7}$ After in

Received: June 2, 2014

Published: July 21, 2014 

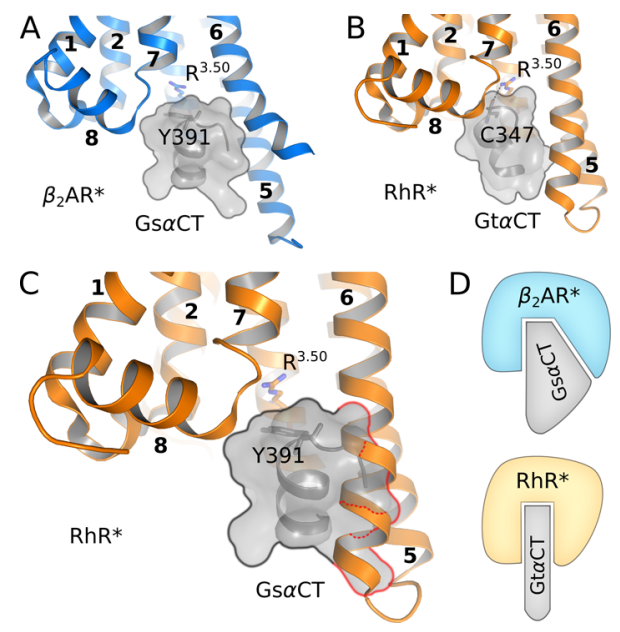

Figure 1. Cytoplasmic crevice of $\beta_{2} \mathrm{AR}^{*}$ (blue) and $\mathrm{RhR}^{*}$ (orange) from complexes with $\mathrm{Gs}^{7}$ and $\mathrm{Gt} \alpha \mathrm{CT}^{6}$ (both in gray ribbon surface representation), respectively. (A-C) Side view: The outward-tilted TM6 of $\beta_{2} \mathrm{AR}^{*}$ allocates space for the bulky Gs $\alpha \mathrm{CT}$ (outlined, transparent surface) that would clash (indicated by the red outline in C) with the more inward-tilted TM6 position of $\mathrm{RhR}^{*}$ (orange) in complex with Gt $\alpha \mathrm{CT}$. $\mathrm{R}^{3.50}$ from the base of the cytoplasmic crevice and $\mathrm{Y} 391 / \mathrm{C} 347$ from the tip of $\mathrm{Gs} / \mathrm{t} \alpha \mathrm{CT}$ are shown as sticks. (D) Schematic representations of the two crystal structure interactions in panels $\mathrm{A}-\mathrm{C}$.
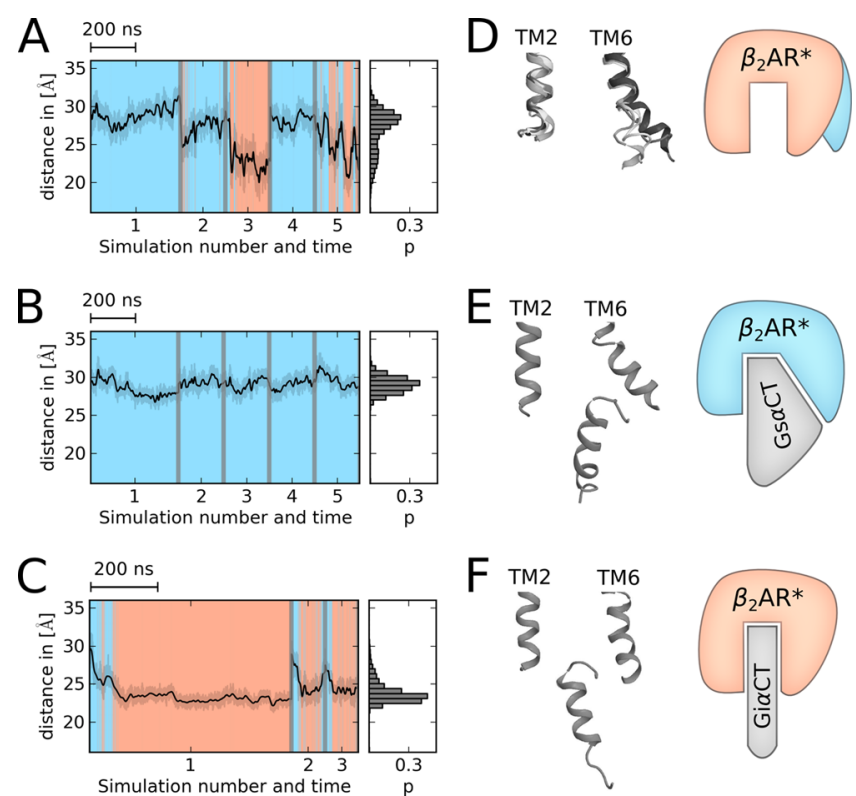

Figure 2. Conformations of the cytoplasmic crevice of $\beta_{2} \mathrm{AR}^{*}$ observed in $\mathrm{MD}$ simulations (A,D) alone, (B,E) with $\mathrm{Gs}_{\alpha} \alpha \mathrm{CT}_{19}$, and $(\mathrm{C}, \mathrm{F})$ with $\mathrm{Gi} \alpha \mathrm{CT}_{19}$. Panels A-C show the time traces of the TM6 tilts $\left(d_{\mathrm{TM} 2-6}\right)$ with resulting distributions and observed probabilities $p$. Panels D-F schematically depict the Gs coupled state (blue) and the $\mathrm{RhR}^{*}$-like state of $\beta_{2} \mathrm{AR}^{*}$ (red) defined by representative TM2 and TM6 conformations. (C) In 3 of 21 simulations (100-600 ns), $\beta_{2} \mathrm{AR}^{*}$ adopts a RhR*-like conformation with Gi $\alpha \mathrm{CT}_{19}$. Crystal structures of $\mathrm{R}$ and $\mathrm{R}^{*}$ exhibit the following TM6 tilts: inactive $\beta_{2} \mathrm{AR}$ (PDB entry 2RH1), 18.2 $\AA$; $\beta_{2} \mathrm{AR}^{*} \cdot \mathrm{Gs}$ (3SN6), $29.7 \AA$; inactive RhR (1U19), 16.4 $\AA$; and $\mathrm{RhR}^{*}$. Gt $\alpha \mathrm{CT}$ (3PQR), $23.2 \AA$.

silico reconstitution of $\beta_{2} \mathrm{AR}^{*}$ into a lipid bilayer, five independent 200-400 ns MD simulations were carried out. In accordance with earlier analyses, ${ }^{10-12}$ we observe a broad distribution of TM6 tilts in the absence of cytoplasmic binding
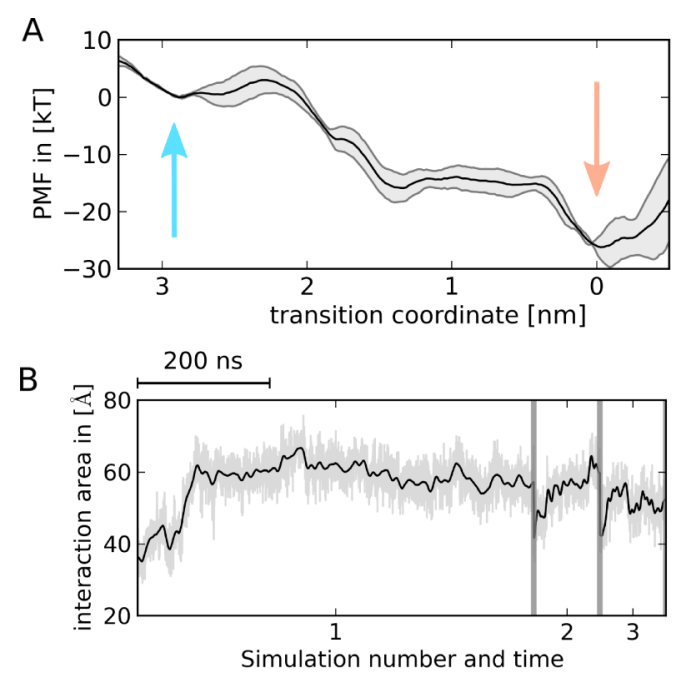

Figure 3. Umbrella sampling run along the coordinates of the TM6 inward movement during transition from the starting state using the receptor coordinates from the $\beta_{2} \mathrm{AR}^{*} \cdot \mathrm{Gs}$ complex to the $\beta_{2} \mathrm{AR}^{*}$. Gi $\alpha \mathrm{CT}_{19}$ complex (see Figure 2C). (A) PMF energy profile (black line) and error estimation (gray area). Arrows indicate the positions of the initial (Gs coupling state, blue) and the final $\beta_{2} \mathrm{AR}^{*}$ conformation (red) on the transition coordinate (see Supporting Information). An energy barrier of $3.0 \pm 2.3 \mathrm{kT}$ is at $2.3 \mathrm{~nm}$ on the transition coordinate, which corresponds to $d_{\mathrm{TM} 2-6} \approx 28.6 \AA$. (B) Increase of the $\beta_{2} \mathrm{AR}^{*} \mathrm{Gi} \alpha \mathrm{CT}_{19}$ interaction surface accompanying the TM6 inward movement.

partners (Figure 2A,D). A pronounced maximum at $d_{\mathrm{TM} 2-6}=$ $28 \AA$ (for definition see SI Methods) reflects that the Gs coupled state is also present in the uncomplexed $\beta_{2} \mathrm{AR}^{*}$. A second peak arises around $23 \AA$, characterizing another population of $\beta_{2} \mathrm{AR}^{*}$ corresponding to the more closed RhR*.Gt $\alpha$ CT state (Figure S1A). Remarkably, during the simulations, TM6 was able to move back and forth between these two states, accompanied by pronounced order-to-disorder transitions of its cytoplasmic end (Figure S1B). This dynamic character is typical for the uncomplexed $\mathrm{R}^{*}$, as has been shown by NMR and FTIR spectroscopy. ${ }^{11,19}$ It is noteworthy that for $\mathrm{RhR}^{*}$, which does not couple to Gs, ${ }^{18}$ larger TM6 outward tilts corresponding to the $\beta_{2} \mathrm{AR}^{*}$. Gs conformation are not observed in $\mathrm{MD}$ simulations of uncomplexed $\mathrm{RhR}^{*}$ (see ref 11 and Figure S2F).

Selective stabilization of a specific $\mathrm{R}^{*}$ conformation out of an ensemble of substates has been suggested as a common mechanism for GPCR-mediated signal transduction. ${ }^{12,19}$ In accordance, the $\beta_{2} \mathrm{AR}^{*}$. Gs complex reconstituted in silico into a lipid bilayer remains essentially unchanged during five $200 \mathrm{~ns}$ simulations, although stabilizing mutations, nanobodies, and the fused T4-lysozyme have been eliminated. TM6 (Figure S2A) and Gs $\alpha$ CT (Figure S3C) are locked in their positions, and the cation $-\pi$ interaction between $\mathrm{Y} 391$ and $\mathrm{R} 131^{3.50}$ of the receptor persists (Figure S4A). In addition, several other polar and hydrophobic interactions, most of them also present in the crystal structure, ${ }^{7}$ fixate Gs $\alpha$ CT to ICL2 and ICL3 (Figure S5A).

$\mathrm{Gs} / \mathrm{i} \alpha \mathrm{CT}$ peptides have been used as surrogates of $\mathrm{G}$ to examine $\mathrm{G}$ protein-selective $\beta_{2} \mathrm{AR}^{*}$ conformations. ${ }^{15,20}$ To identify the minimum peptide length required for complex stabilization, we truncated Gs to a Gs $\alpha \mathrm{CT} 19$-mer (Gs $\alpha \mathrm{CT}_{19}$, ${ }^{376}$ FNDCRDIIQRMHLRQYELL ${ }^{394}$ ). Gs $\alpha \mathrm{CT}_{19}$ (Figure S3) and TM6 (Figures 2B) remain in their conformations during 
A

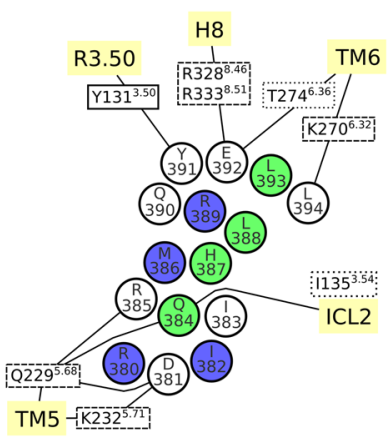

$\beta 2 \mathrm{AR}^{*} \cdot \mathrm{Gs} \alpha \mathrm{CT}$

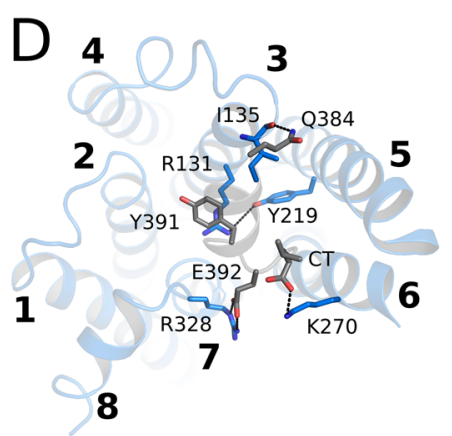

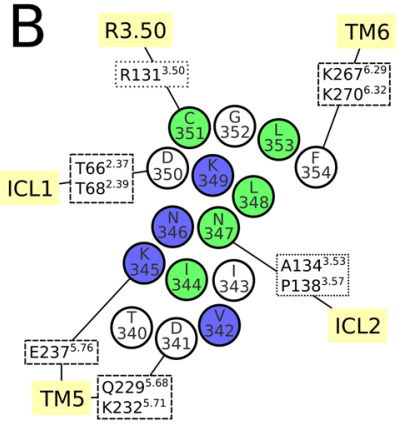

$\beta_{2} A R^{*} \cdot G i \alpha C T$

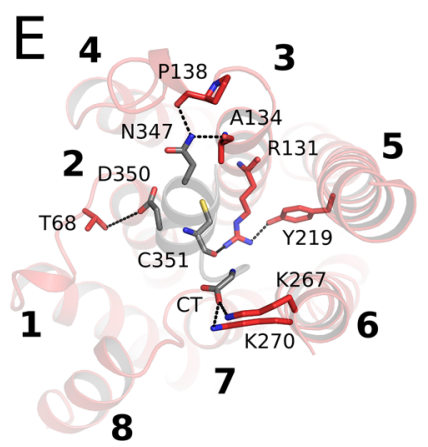

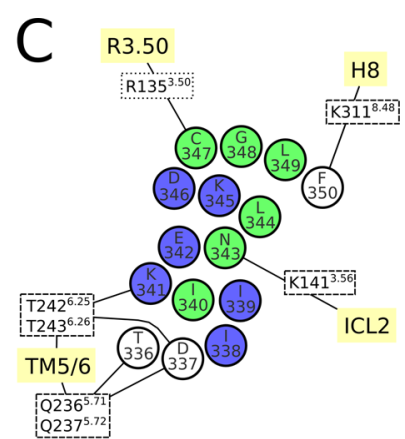

$\mathrm{RhR}^{*} \cdot \mathrm{Gt} \alpha \mathrm{CT}$

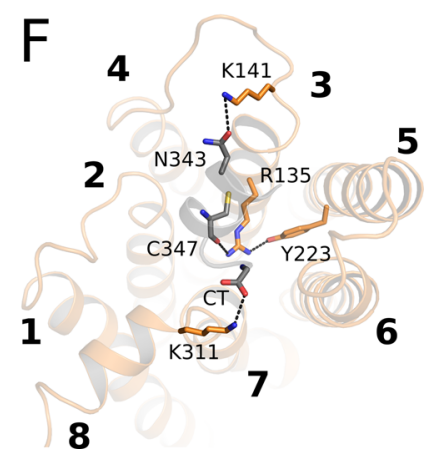

Figure 4. Contacts observed in $\mathrm{MD}$ simulations of various $\mathrm{R}^{*} \cdot \mathrm{G} \alpha \mathrm{CT}_{19}$ complexes. (A-C) Recurring polar contacts involving side chains (dashed boxes), backbone carbonyl (dotted boxes), or cation $-\pi$ interactions (solid boxes) between peptides (circles) and receptors (boxes) depicted as interaction schemes. Buried residues are colored green; accessible residues are blue (see also Figure S8). (D-E) Top view from the intracellular side on the different $\mathrm{R}^{*} \cdot \mathrm{G} \alpha \mathrm{CT}_{19}$ complexes. Hydrogen bonds between residues (sticks) of peptides (gray) and $\mathrm{R}^{*}$ (colored) are indicated as dotted lines. For clarity, only the C-terminal 11 residues of the $\mathrm{G} \alpha \mathrm{CT}_{19}$ peptides are shown in panels $\mathrm{D}-\mathrm{F}$, but the full set of interactions is described in panels A-C.

all simulations, stabilized by the same specific interactions as with Gs (Figures S4B and S5B). Further N-terminal truncation to $\mathrm{Gs} \alpha \mathrm{CT}_{11}$ reduces the number of specific contacts with $\beta_{2} \mathrm{AR}^{*}$ (Figures S4C and S5C), leading to a higher positional variability of Gs $\alpha \mathrm{CT}_{11}$ (Figure S3) and TM6 (Figure S2). Our simulations suggest that the binding interface for Gs coupling to $\beta_{2} \mathrm{AR}^{*}$ consists of $15 \mathrm{C}$-terminal residues (see Figures $4 \mathrm{~A}$, S4A-C, and S5A-C) which form a scaffold between ICL2 and TM5/6, stabilizing the more open cytoplasmic crevice of the Gs coupled receptor. Notably, in simulations of a putative RhR*.Gs $\alpha \mathrm{CT}$ peptide complex (see SI Methods), the characteristic cation $-\pi$ interaction between Y391 and $\mathrm{R} 131^{3.50}$ does not form as with $\beta_{2} \mathrm{AR}^{*} \cdot \mathrm{Gs} \alpha \mathrm{CT}$ (Figure S4), in accordance with the absence of a Gs signaling pathway in the visual RhR system. ${ }^{21}$

Subsequently, we addressed the question whether a Gi $\alpha$ Cterminal 19-mer ( $\mathrm{Gi} \alpha \mathrm{CT}_{19},{ }^{336}$ FDAVTDVIIKNNLKD$\mathrm{CGLF}^{354}$ ) can stabilize the RhR*-like conformation observed for the uncomplexed $\beta_{2} \mathrm{AR}^{*}$. The starting position of Gi $\alpha \mathrm{CT}_{19}$ was extrapolated from the crystal structure complex of Gt $\alpha \mathrm{CT}_{19} \quad\left({ }^{332} \mathrm{FDAVTDIIIKENLKDCGLF}^{350}\right)$ with $\mathrm{RhR}^{*}{ }^{6}$ The receptor coordinates were taken from the $\beta_{2} \mathrm{AR}^{*} \cdot \mathrm{Gs}$ crystal structure complex. ${ }^{7}$ In this starting configuration Gi $\alpha \mathrm{CT}_{19}$ does not have any contact with TM6. Similar to the simulations of uncomplexed $\beta_{2} \mathrm{AR}^{*}$, TM6 tends to persist in its starting position within the first hundreds of nanoseconds (Figure S2E). However, in 3 out of 21 independent simulations, the initial receptor conformation is left, and TM6 tilts inward spontaneously by $6 \AA$ (Figure 2C).

To obtain information about the energy barrier that retards formation of the more closed $\beta_{2} \mathrm{AR}^{*}$ state bound to $\mathrm{Gi} \alpha \mathrm{CT}$
$\left(d_{\mathrm{TM} 2-6}=23 \AA\right)$, we performed umbrella sampling (US) MD simulations along one of these trajectories and calculated the potential of mean force (PMF, Figure 3A). The PMFs show that such an energy barrier exists, arising from the reorganization of interactions between TM5/6 (Figure S6B). As soon as this barrier is overcome, the TM6 inward movement follows a continuous downhill reaction. The interaction surface with $\mathrm{Gi} \alpha \mathrm{CT}_{19}$ that remains at its starting position increases (Figure 3B), and the key interaction between C351 and $\mathrm{R} 131^{3.50}$ forms. This key interaction and a hydrogen bond between N347 and P138 $8^{3.57}$ from the second intracellular loop (ICL2) and from D341 and K345 to ICL3 are also observed in $\mathrm{MD}$ simulations of Gt $\alpha \mathrm{CT}$ with $\mathrm{RhR}^{*}$ (Figures $4 \mathrm{C}$ and S5E), supporting the notion that $\beta_{2} \mathrm{AR}^{*}$ forms a complex with Gi $\alpha \mathrm{CT}$ very similar to the complex RhR* forms with Gt $\alpha \mathrm{CT}$ (Figure S7C).

The $\alpha$-helical content of TM6 increases upon complex formation (Figure S6A), in accordance with our recently proposed model of receptor $G$ protein coupling through structural stabilization of the binding crevice. ${ }^{11}$ Extrapolation of the $\mathrm{Gi} \alpha \mathrm{CT}_{19}$ peptide to the $\mathrm{Gi}$ holoprotein in a putative $\beta_{2} \mathrm{AR}^{*} \cdot \mathrm{Gi} \alpha \beta \gamma$ complex (model based on $\beta_{2} \mathrm{AR}^{*} \cdot \mathrm{Gs}^{7}$ ) results in a feasible arrangement without clashes (Figure S7A). Finally, our analysis of $\mathrm{Gi}$ vs $\mathrm{Gs}$ interactions explains previous mutational data, in which a chimeric Gs $\alpha / \mathrm{Gt} \alpha$ was capable of binding to and being potently activated by $\mathrm{RhR}^{*}{ }^{22}$ The two Gs $\alpha$ triple mutants that made Gs $\alpha$ light-activatable were indeed exchanged at those amino acid positions, where we observe differential interactions of $\mathrm{Gs}$ and $\mathrm{Gi}$, namely $\mathrm{Y} 391$ to $\mathrm{R}^{3.50}$, E392 to TM6/H8 for Gs (Figure 4A) or N347 with ICL2, C351 with $\mathrm{R}^{3.50}$, and D350 with ICL1 for Gi (Figure 4B). 
In accordance with earlier studies, ${ }^{3,12,19}$ we observe a strong structural heterogeneity for the two active GPCRs, $\beta_{2} \mathrm{AR}^{*}$ and $\mathrm{RhR}^{*}$, reflected by broad distributions of different TM6 tilts in the absence of intracellular interaction partners. In the case of $\beta_{2} \mathrm{AR}^{*}$, this distribution exhibits at least two pronounced maxima at 23 and $28 \AA$. The maximum at $28 \AA$ represents the Gs coupled state, ${ }^{7}$ while the maximum at $23 \AA$ indicates a second $\beta_{2} \mathrm{AR}^{*}$ conformation very similar to the one observed in the Gt $\alpha \mathrm{CT} \cdot \mathrm{RhR}^{*}$ complex. ${ }^{6} \mathrm{Gi} \alpha \mathrm{CT}$, a close homologue of $\mathrm{Gt} \alpha \mathrm{CT}$, stabilizes that more closed $\beta_{2} \mathrm{AR}^{*}$ conformation while-due to its bulkiness-Gs $\alpha \mathrm{CT}$ requires the more open cytoplasmic crevice with a much larger TM6 outward tilt for binding. These observations suggest that $\mathrm{Gs}$ and $\mathrm{Gi} / \mathrm{t}$ have different binding modes, both imprinting their own shape onto the cytoplasmic $\mathrm{R}^{*}$ crevice through specific interactions of $\mathrm{G} \alpha \mathrm{CT}$ with $\beta_{2} \mathrm{AR}^{*}$.

Our results extend an emerging concept of GPCR signaling based on multiple receptor conformations in equilibrium, ${ }^{12}$ each exhibiting specific affinities to the variety of extracellular ligands and intracellular proteins. ${ }^{2,23,24}$ In the example studied here, the $\mathrm{G} \alpha \mathrm{C}$-termini of $\mathrm{Gs}$ or $\mathrm{Gi}$, representing the key sites for interactions with the active $\beta_{2} \mathrm{AR}^{*}$, are able to select and stabilize specific active conformations from a pre-existing equilibrium of agonist-bound receptor states (Figure 2). Thus, the receptor provides a characteristic amount of TM6 flexibility, while the $\mathrm{G} \alpha$ C-terminus selects a specific conformation for productive interaction and signal transfer by presenting a surprisingly small number of crucial residues (Figure 4 and ref 22). Taken together, the abundance of a specific $\mathrm{R}^{*}$ conformation, in addition to the availability and affinity of different $G$ proteins, co-determines which pathway prevails in the intracellular network.

\section{ASSOCIATED CONTENT}

\section{S Supporting Information}

Preparation and execution of MD simulations and US calculations, performed and analyzed with GROMACS; GPCRs were selected on the basis of the availability of an Xray structure of a receptor $G$ protein (or peptides derived thereof) complex, which is a prerequisite for sufficiently accurate atomistic simulations. This material is available free of charge via the Internet at http://pubs.acs.org.

\section{AUTHOR INFORMATION}

\section{Corresponding Author}

peter.hildebrand@charite.de

\section{Present Address}

${ }^{\nabla}$ M.E.: Jules Stein Eye Institute and Department of Chemistry and Biochemistry, University of California, Los Angeles

\section{Notes}

The authors declare no competing financial interest.

\section{ACKNOWLEDGMENTS}

This work was supported by the Deutsche Forschungsgemeinschaft Sfb740 (to K.P.H. and P.W.H.), Sfb1078-B6 (to P.S.), DFG Cluster of Excellence "Unifying Concepts in Catalysis" (Research Field D3/E3-1 to P.S.), HI 1502/1-1 (to P.W.H.), BI 893/8 (to P.W.H.), DFG research fellowship (EL779 to M.E.), and ERC Advanced grant (ERC-2009/249910-TUDOR to K.P.H.). The computer time necessary for this project was provided in part by the "Norddeutscher Verbund für Hochund Höchstleistungsrechner" (HLRN).

\section{REFERENCES}

(1) Pierce, K. L.; Premont, R. T.; Lefkowitz, R. J. Nat. Rev. Mol. Cell. Biol. 2002, 3, 639.

(2) Wisler, J. W.; Xiao, K.; Thomsen, A. R. B.; Lefkowitz, R. J. Curr. Opin. Cell Biol. 2014, 27, 18.

(3) Liu, J. J.; Horst, R.; Katritch, V.; Stevens, R. C.; Wüthrich, K. Science 2012, 335, 1106.

(4) Xiao, R. P. Sci. STKE 2001, 2001, re15.

(5) Altenbach, C.; Kusnetzow, A. K.; Ernst, O. P.; Hofmann, K. P.; Hubbell, W. L. Proc. Natl. Acad. Sci. U.S.A. 2008, 105, 7439.

(6) Scheerer, P.; Park, J. H.; Hildebrand, P. W.; Kim, Y. J.; Krauss, N.; Choe, H. W.; Hofmann, K. P.; Ernst, O. P. Nature 2008, 455, 497.

(7) Rasmussen, S. G. F.; DeVree, B. T.; Zou, Y.; Kruse, A. C.; Chung, K. Y.; Kobilka, T. S.; Thian, F. S.; Chae, P. S.; Pardon, E.; Calinski, D.; Mathiesen, J. M.; Shah, S. T. A.; Lyons, J. A.; Caffrey, M.; Gellman, S. H.; Steyaert, J.; Skiniotis, G.; Weis, W. I.; Sunahara, R. K.; Kobilka, B. K. Nature 2011, 477, 549.

(8) Kruse, A. C.; Ring, A. M.; Manglik, A.; Hu, J.; Hu, K.; Eitel, K.; Hübner, H.; Pardon, E.; Valant, C.; Sexton, P. M.; Christopoulos, A.; Felder, C. C.; Gmeiner, P.; Steyaert, J.; Weis, W. I.; Garcia, K. C.; Wess, J.; Kobilka, B. K. Nature 2013, 504, 101.

(9) Xu, F.; Wu, H.; Katritch, V.; Han, G. W.; Jacobson, K. a; Gao, Z. G.; Cherezov, V.; Stevens, R. C. Science 2011, 332, 322.

(10) Dror, R. O.; Arlow, D. H.; Maragakis, P.; Mildorf, T. J.; Pan, A. C.; Xu, H.; Borhani, D. W.; Shaw, D. E. Proc. Natl. Acad. Sci. U.S.A. 2011, 108, 18684.

(11) Elgeti, M.; Rose, A. S.; Bartl, F. J.; Hildebrand, P. W.; Hofmann, K. P.; Heck, M. J. Am. Chem. Soc. 2013, 135, 12305.

(12) Nygaard, R.; Zou, Y.; Dror, R. O.; Mildorf, T. J.; Arlow, D. H.; Manglik, A.; Pan, A. C.; Liu, C. W.; Fung, J. J.; Bokoch, M. P.; Thian, F. S.; Kobilka, T. S.; Shaw, D. E.; Mueller, L.; Prosser, R. S.; Kobilka, B. K. Cell 2013, 152, 532

(13) Hamm, H. E.; Deretic, D.; Arendt, A.; Hargrave, P. A.; Koenig, B.; Hofmann, K. P. Science 1988, 241, 832.

(14) Kisselev, O. G.; Kao, J.; Ponder, J. W.; Fann, Y. C.; Gautam, N.; Marshall, G. R. Proc. Natl. Acad. Sci. U.S.A. 1998, 95, 4270.

(15) Oldham, W. M.; Hamm, H. E. Nat. Rev. Mol. Cell. Biol. 2008, 9, 60

(16) Choe, H.-W.; Kim, Y. J.; Park, J. H.; Morizumi, T.; Pai, E. F.; Krauss, N.; Hofmann, K. P.; Scheerer, P.; Ernst, O. P. Nature 2011, 471,651 .

(17) Standfuss, J.; Edwards, P. C.; D’Antona, A.; Fransen, M.; Xie, G.; Oprian, D. D.; Schertler, G. F. X. Nature 2011, 471, 656.

(18) Cerione, R. A.; Staniszewski, C.; Benovic, J. L.; Lefkowitz, R. J.; Caron, M. G.; Gierschik, P.; Somers, R.; Spiegel, A. M.; Codina, J.; Birnbaumer, L. J. Biol. Chem. 1985, 260, 1493.

(19) Kim, T. H.; Chung, K. Y.; Manglik, A.; Hansen, A. L.; Dror, R. O.; Mildorf, T. J.; Shaw, D. E.; Kobilka, B. K.; Prosser, R. S. J. Am. Chem. Soc. 2013, 135, 9465.

(20) Malik, R. U.; Ritt, M.; DeVree, B. T.; Neubig, R. R.; Sunahara, R. K.; Sivaramakrishnan, S. J. Biol. Chem. 2013, 288, 17167.

(21) Hepler, J. R.; Gilman, A. G. Trends Biochem. Sci. 1992, 17, 383.

(22) Natochin, M.; Muradov, K. G.; McEntaffer, R. L.; Artemyev, N. O. J. Biol. Chem. 2000, 275, 2669.

(23) Rahmeh, R.; Damian, M.; Cottet, M.; Orcel, H.; Mendre, C.; Durroux, T.; Sharma, K. S.; Durand, G.; Pucci, B.; Trinquet, E.; Zwier, J. M.; Deupi, X.; Bron, P.; Banères, J.-L.; Mouillac, B.; Granier, S. Proc. Natl. Acad. Sci. U.S.A. 2012, 109, 6733.

(24) Soriano-Ursúa, M. A.; Trujillo-Ferrara, J. G.; Correa-Basurto, J.; Vilar, S. J. Med. Chem. 2013, 56, 8207. 